\title{
Las Funciones Semióticas como Instrumento de Diagnóstico y Abordaje de Errores
}

\author{
Semiotics Functions as a Tool for Diagnosis and Error Approach
}

\author{
Andrea Aznar* \\ Sandra Baccelli ${ }^{* *}$ \\ Stella Figueroa ${ }^{* *}$ \\ María Laura Distéfano**
}

Sergio Anchorena ${ }^{* * * *}$

\begin{abstract}
Resumen
Las funciones semióticas constituyen herramientas teóricas y metodológicas utilizadas por el Enfoque Ontosemiótico de la Cognición y la Instrucción Matemática (EOS) para el estudio de la construcción de significados de objetos matemáticos. En este trabajo, a través de algunos ejemplos, se presenta la descripción del uso de tales funciones para el abordaje de distintas problemáticas de aprendizaje en estudiantes universitarios. A partir de los antecedentes y ejemplos expuestos, puede observarse el potencial que estas herramientas tienen para el diagnóstico y tratamiento de distintos tipos de errores asociados a prácticas matemáticas. Las funciones semióticas permiten: visibilizar el nivel de complejidad de una determinada práctica matemática; evaluar detalladamente prácticas matemáticas de estudiantes para detectar discrepancias de significados, y favorecer el rediseño de secuencias didácticas, contemplando las complejidades y conflictos detectados. También se exponen los pasos de refinamientos sucesivos para la definición de las funciones semióticas apropiadas para el análisis de algún problema particular. De esta manera, se muestra una sistematización de este proceso que posibilita su aplicación en otras investigaciones referidas al estudio de significados.
\end{abstract}

Palabras-clave: Funciones semióticas. Significado. Herramienta metodológica. Enfoque Ontosemiótico.

\footnotetext{
* Especialista en Investigación Educativa por la Universidad Nacional de Tucumán (UNT). Docente e investigadora en la Facultad de Ingeniería de la Universidad Nacional de Mar del Plata (UNMDP), Mar del Plata, Buenos Aires, Argentina. Dirección postal: Juan B. Justo 2002, (7600), Mar del Plata, Buenos Aires, Argentina. E-mail: maznar@fi.mdp.edu.ar

** Especialista en Investigación Educativa por la Universidad Nacional de Tucumán (UNT). Docente e investigadora en la Facultad de Ingeniería de la Universidad Nacional de Mar del Plata (UNMDP). Mar del Plata, Buenos Aires, Argentina. Dirección postal: Juan B. Justo 2002, (7600) Mar del Plata, Buenos Aires, Argentina. E-mail: sbaccelli@gmail.com

**** Magíster en Enseñanza de la Matemática en el Nivel Superior por la Universidad Nacional de Tucumán (UNT). Docente e investigadora en la Facultad de Ingeniería de la Universidad Nacional de Mar del Plata (UNMDP). Mar del Plata, Buenos Aires, Argentina. Dirección postal: Juan B. Justo 2002, (7600) Mar del Plata, Buenos Aires, Argentina. E-mail: stellafigueroa@gmail.com

Magíster en Enseñanza de la Matemática en el Nivel Superior por la Universidad Nacional de Tucumán (UNT). Docente e investigadora en la Facultad de Ingeniería de la Universidad Nacional de Mar del Plata (UNMDP). Mar del Plata, Buenos Aires, Argentina. Dirección postal: Juan B. Justo 2002, (7600) Mar del Plata, Buenos Aires, Argentina. E-mail: mldistefano@fi.mdp.edu.ar

****** Doctor en Filosofía y Ciencias de la Educación por la Universidad del País Vasco (UPV). Docente e investigador en la Facultad de Cs. de la Salud y Servicio Social. Facultad de Humanidades. Universidad Nacional de Mar del Plata (UNMDP). Mar del Plata, Buenos Aires, Argentina. Dirección postal: Funes 3350, Acceso 1, Cuerpo 2, Nivel 4, (7600) Mar del Plata, Buenos Aires, Argentina. E-mail: pollo_mdp@yahoo.com
} 


\begin{abstract}
Semiotic functions are theoretical and methodological tools used by the Ontosemiotic Approach of Cognition and Mathematics Instruction (OSA) to study the construction of meanings of mathematical objects. In this paper, through some examples, the use of such functions is described for dealing different problems in university students learning. From the background and the stated examples, it can be seen that these tools have potential for the diagnosis and treatment of many kinds of errors associated with mathematical practices. Semiotic functions allow: visualization of the level of complexity of a particular mathematical practice; accurately assessment of the student mathematical practice for meaning discrepancies, and promotion of the redesign of teaching sequences, bearing in mind the complexities and conflicts detected. It is also outlined the steps of successive refinements for defining appropriate semiotic functions for the analysis of a particular problem. Thus, a systematization of this process is shown, which enables its application in other researches related to the study of meanings.
\end{abstract}

Keywords: Semiotic functions. Meaning. Methodological tool. Ontosemiotic Approach.

\title{
1 Introducción
}

Gran parte de las investigaciones de Educación Matemática abordan el problema de dificultades frecuentes que enfrentan los estudiantes ante determinadas prácticas matemáticas. Desde diferentes marcos teóricos se proponen variadas herramientas que permiten el análisis de dichas prácticas con la intención de explicar esas dificultades y proponer posibles estrategias tendientes a su superación.

El interés de realizar un análisis de errores en el aprendizaje ha sido señalado por diversos autores, quienes piensan que son una parte inseparable de este proceso (RADATZ, 1980; BORASSI, 1987; RICO, 1995; POCHULU, 2004). A su vez, el interés radica en la posibilidad de caracterizar las regularidades con que se presentan los errores y de construir modelos explicativos, pues es una estrategia valiosa para clarificar dificultades en el aprendizaje matemático y permitiría plantear propuestas superadoras.

En este trabajo utilizamos el Enfoque Ontosemiótico del Conocimiento y la Instrucción Matemática (EOS) (GODINO; BATANERO, 1994; GODINO; CONTRERAS; FONT, 2006; GODINO; BATANERO; FONT, 2009; POCHULU, 2012). En particular, nos centraremos en una de las herramientas teórico-metodológicas propuestas por este enfoque, la función semiótica, entendida como una entidad que asigna una relación específica entre objetos matemáticos. Con dicha herramienta se procura “...modelizar la actividad matemática, tanto en los procesos de resolución de problemas, como en los procesos comunicativos en la enseñanza y aprendizaje, como secuencias de funciones semióticas e identificar conflictos semióticos potenciales o efectivos en la interacción didáctica" (GODINO, 2003, p.145). 
Se presenta parte de la experiencia del grupo GIEMI ${ }^{1}$, en las que se analizan procesos de resolución de problemas matemáticos, estableciendo la secuencia de funciones semióticas implicadas en ese proceso. Dichas funciones son relevantes tanto en su contenido como en su orden, ya que, en caso de no establecerse correctamente, no se logra resolver satisfactoriamente el problema en cuestión.

En este artículo se presentan funciones semióticas asociadas a la resolución de problemas de tipos específicos, utilizadas en cuatro trabajos del grupo, con su correspondiente fundamentación, a fin de compartir la experiencia y la metodología con otros investigadores. En dichos trabajos se describen algunas de las dificultades que se generan en los procesos de enseñanza y de aprendizaje de distintos objetos matemáticos vinculados a: problemas de optimización, representaciones de números complejos, representaciones simbólicas en el registro algebraico y problemas asociados a la probabilidad condicional. Mediante el análisis de las funciones semióticas implicadas, se determinan los significados personales asignados a los objetos matemáticos involucrados, y se las contrasta con las funciones semióticas que representan los significados pretendidos. Dicho análisis ha permitido:

- Identificar los conflictos de significados que pueden obstaculizar el aprendizaje en un proceso de instrucción.

- Obtener información relevante para el diseño de estrategias de enseñanza.

En los procesos de definición de las funciones semióticas de los cuatro trabajos se hallaron algunas regularidades. Las mismas se enuncian, esperando que sirvan como criterios para ser considerados en estudios similares.

\section{Antecedentes}

Con miras al estudio de los factores que intervienen en la generación de obstáculos o dificultades en el aprendizaje de la matemática se han realizado distintas clasificaciones de los errores.

Así, Radatz (1980, citado en ABRATE; POCHULU; VARGAS, 2006) señala una categorización según la causa:

(a) Dificultades del lenguaje; (b) Dificultades para obtener información espacial; (c) Aprendizaje deficiente de hechos, destrezas y conceptos previos; (d) Asociaciones incorrectas

\footnotetext{
1 Grupo de Investigación: Enseñanza de la Matemática en Carreras de Ingeniería (GIEMI), Facultad de Ingeniería Universidad Nacional de Mar del Plata, Argentina.
} 
o rigidez del pensamiento (por perseveración, de asociación, de interferencia o de asimilación); (e) Aplicación de reglas o estrategias irrelevantes.

Asimismo, Mavshovitz-Hadar, Zaslavksy e Invar (citados en Rico, 1995) plantean categorías empíricas y descriptivas de los errores, sobre la base de un análisis constructivo de las soluciones de los alumnos realizadas por expertos: (a) Datos mal utilizados; (b) Interpretación incorrecta del lenguaje; (c) Inferencias no válidas lógicamente; (d) Teoremas o definiciones deformados; (e) Falta de verificación en la solución; (f) Errores técnicos.

El enfoque pragmático que tiene el EOS asocia los significados de los objetos matemáticos a las prácticas y dichas prácticas se representan mediante funciones semióticas de distinto tipo. En este contexto, los errores se identifican con la falta de establecimiento de las funciones semióticas o con el establecimiento de funciones semióticas incorrectas.

Existen antecedentes de trabajos en los que se utilizan funciones semióticas como herramientas metodológicas, focalizados sobre los procesos de enseñanza y de aprendizaje de distintos contenidos matemáticos. A continuación, se describen sintéticamente dichos trabajos.

Contreras et al. (2005) realizan un análisis semiótico de un texto en el que se define la función derivada. Realizan dicho análisis considerando como expresión o contenido de las funciones semióticas que definen, las facetas duales extensiva - intensiva de los objetos matemáticos presentes en las unidades de análisis consideradas en el texto. A través del análisis detectan conflictos semióticos potenciales y focalizan la atención, a través de las facetas duales mencionadas, sobre el uso de elementos genéricos, cruciales en la actividad matemática. Una tarea similar realizan Contreras y Ordoñez (2006) al analizar la introducción a integral definida presentada en un fragmento de un texto de bachillerato. A través de la definición de funciones semióticas que contemplan las dualidades de los objetos matemáticos relacionados, describen la complejidad de la comprensión del mismo y anticipan posibles conflictos semióticos.

Chacón (2006) utiliza funciones semióticas para hacer un análisis de una trayectoria epistémica de un proceso de enseñanza de la definición algebraica de estructura de grupo a desarrollarse en un contexto virtual. Identifica unidades de análisis en dicha trayectoria para luego asociarlas a funciones semióticas. Éstas últimas son clasificadas según la tabla propuesta por Font (2002). Análogamente, define unidades de análisis y sus funciones semióticas asociadas en una tarea dada a un estudiante. Dichas funciones también son clasificadas según la tabla mencionada.

Tamayo et al. (2008) analizan un proceso de enseñanza y aprendizaje de la 
definición de Límite funcional según Weierstrass, implementado en una institución escolar en un ambiente de tecnología digital; descomponen dicho proceso en unidades para identificar entidades y las funciones semióticas que se establecen. Identifican nueve tipos de funciones semióticas cuyas expresiones o contenidos son notacionales o facetas de la dualidad intensivo-extensivo.

Godino, Batanero y Roa (2005) estudian el proceso de resolución de algunos problemas combinatorios por alumnos con alta preparación matemática, aportando una explicación semiótica del razonamiento combinatorio y describiendo las implicaciones del modelo teórico. Afirman que los objetos matemáticos no son entidades aisladas, sino que se ponen en relación en el lenguaje y la actividad matemática por medio de funciones semióticas. Es decir, las conexiones entre un antecedente (expresión, significante) y un consecuente (contenido o significado), establecidas por un sujeto (persona o institución) de acuerdo con un cierto criterio de correspondencia. Consideran, en los ejemplos que desarrollan, que cualquiera de los objetos matemáticos puede desempeñar el rol de expresión y contenido. En los ejemplos analizados se determinan los conflictos semióticos, pero no se definen explícitamente las funciones semióticas intervinientes en esa disparidad de significados. Sugieren, finalmente, sobre la utilidad en considerar un razonamiento matemático como secuencia de funciones semióticas (o cadena de conocimientos puestos en relación) en el proceso de resolución de un problema por parte de un sujeto.

Resulta interesante el uso teórico-metodológico de las funciones semióticas realizado por Rojas Garzón (2012). En su tesis estudia las dificultades que encuentran algunos estudiantes para articular los sentidos asignados a representaciones semióticas de un mismo objeto matemático, obtenidas mediante tratamiento. Utiliza las funciones semióticas para definir el sentido de un objeto matemático primario como el contenido de la función semiótica que tiene a dicho objeto primario como expresión de la función semiótica. A partir de esta noción de sentido, define la articulación de sentidos como el establecimiento de una función semiótica resultante de la concatenación de dos funciones semióticas: uno de los sentidos (contenido) del objeto primario se convierte en expresión de una nueva función semiótica que tiene como contenido a otro sentido de dicho objeto. Asimismo, usa el constructo función semiótica para definir la actividad cognitiva de tratamiento, afirmando que "el tratamiento se puede caracterizar como una función semiótica que toma como equivalentes dos objetos primarios, considerados ambos como representaciones de un mismo objeto matemático" (ROJAS GARZÓN, 2012, p.53).

En su trabajo presenta la configuración cognitiva de objetos matemáticos primarios de 
producciones de estudiantes en el desarrollo de tareas de probabilidad, triple de un número y sobre cónicas, resaltando las funciones semióticas establecidas por éstos en su proceso de significación. Presenta evidencias que confirman los obstáculos que encuentran los estudiantes para articular sentidos.

\section{Marco teórico}

El marco teórico en el que se fundamentan los análisis es el Enfoque Ontosemiótico de la Cognición y la Instrucción Matemática (EOS), como línea de investigación en Didáctica de la Matemática, que viene desarrollándose en España desde el año 1994 por Juan Díaz Godino, Carmen Batanero y Vicenç Font Moll (GODINO; BATANERO, 1994; GODINO; CONTRERAS; FONT, 2006; GODINO; BATANERO; FONT, 2009; POCHULU, 2012).

Este enfoque define a una práctica matemática como cualquier acción, expresión o manifestación (lingüística o de otro tipo) realizada por alguien para resolver problemas matemáticos, comunicar la solución obtenida a otras personas, validar y generalizar esa solución a otros contextos, A partir de este constructo, surge la noción de significado, definido como el sistema de prácticas operativas y discursivas para resolver un cierto tipo de problemas (GODINO; BATANERO; FONT, 2009).

En el contexto de una clase, los conocimientos de cada alumno en un momento dado son muy variados. Ante un determinado objeto matemático se considera el significado personal que cada alumno le asigna a dicho objeto para diferenciarlo del significado fijado por el profesor, por el libro de texto o en un currículo (significado institucional).

A partir de esta distinción se puede describir, metafóricamente, el aprendizaje como acoplamiento progresivo entre significados personales e institucionales en una clase. Esta diferenciación entre las facetas personal e institucional de los significados matemáticos es fundamental para poder describir y explicar las interacciones entre el profesor y los estudiantes en los procesos de enseñanza y aprendizaje (GODINO; BATANERO; FONT, 2009).

En los sistemas de prácticas matemáticas intervienen y aparecen conjuntos de objetos conformando redes llamadas configuraciones. Las mismas pueden ser cognitivas o epistémicas de acuerdo la condición de personal o institucional de los objetos involucrados. Estas configuraciones, junto con los sistemas de prácticas, son las herramientas teóricas que propone este enfoque para describir los conocimientos matemáticos expresados en términos de significados (GODINO; BATANERO; FONT, 2009). 
Para describir la actividad matemática y los procesos cognitivos implicados, el EOS considera la teoría del lenguaje de Hjemslev (1943, citado en GODINO, 2003), quien denomina función a la dependencia entre el texto y sus componentes y entre estos componentes entre sí. Entre los tipos de dependencias que es posible identificar entre las partes de un texto, resaltan aquellas en que una parte (plano de expresión) se pone en representación de otra (plano del contenido). Esta función es la que Hjemslev designa función de signo y que Eco presenta como función semiótica (GODINO, 2003).

Las funciones semióticas ponen de manifiesto la naturaleza esencialmente relacional de la actividad matemática. Godino (2003) considera que esa dependencia no es sólo representacional, sino que existen otras dependencias de naturaleza operatoria o actuativa.

En este contexto, el significado está conformado por una trama de funciones semióticas, cada una de las cuales asigna a una expresión un contenido, mediante un cierto criterio o regla de correspondencia establecida por un sujeto. Este contenido puede ser un término, expresión, gráfico u otro elemento lingüístico, una situación problema, un concepto o definición, una acción u operación, algoritmo o procedimiento, una argumentación, etc. (GODINO; BATANERO; FONT, 2009). Es decir que el contenido puede ser cualquiera de los objetos primarios ${ }^{2}$ que el EOS contempla en su ontología. Esto permite clasificar las funciones semióticas según los tipos que se presentan en Cuadro 1. Esta es la clasificación utilizada en los ejemplos de aplicación que se presentan en la próxima sección.

\begin{tabular}{lc}
\hline Tipo de función semiótica & Contenido \\
\hline Lingüística & Término, expresión, gráfico u otro elemento \\
lingüístico
\end{tabular}

Cuadro 1- Tipos de funciones semióticas Fuente: Pochulu (2012)

Las funciones semióticas poseen un gran potencial como herramientas metodológicas al momento de estudiar significados, particularmente los significados personales construidos por los estudiantes. Font (2002, p.156) expresa que:

El hecho de considerar que las funciones semióticas tienen un papel muy importante

\footnotetext{
${ }^{2}$ Los objetos primarios considerados por el EOS son: Situaciones, Lenguaje, Definiciones, Proposiciones, Procedimientos y Argumentos (GODINO; BATANERO; FONT, 2009)
} 
en el proceso relacional entre entidades (o grupos de ellas), activadas en prácticas que se realizan dentro de un determinado juego de lenguaje, permite también entender la comprensión en términos de funciones semióticas. En efecto, podemos interpretar la comprensión de un objeto $\mathrm{O}$ por parte de un sujeto $\mathrm{X}$ (sea individuo o institución) en términos de funciones semióticas que $\mathrm{X}$ puede establecer, en unas circunstancias fijadas, en las que se pone en juego $\mathrm{O}$ como funtivo (expresión o contenido).

La falta de establecimiento de los distintos tipos de funciones semióticas, o el establecimiento de funciones incorrectas, da lugar a errores que pueden ubicarse en alguna de las clasificaciones mencionadas en el apartado anterior.

Así por ejemplo, las dificultades provocadas por la falta de establecimiento de funciones semióticas lingüísticas pueden asociarse a la categoría errores causado por Dificultades del lenguaje, de Radatz (1980, citado en ABRATE; POCHULU; VARGAS, 2006) o a la categoría Interpretación incorrecta del lenguaje, de Mavshovitz-Hadar, Zaslavksy e Invar (citados en RICO, 1995). Los errores asociados a las categorías de Mavshovitz-Hadar, Zaslavksy e Invar denominadas Datos mal utilizados y Falta de verificación en la solución se corresponden con la ausencia o establecimiento deficiente de funciones semióticas situacionales y argumentativas respectivamente.

Más allá de tipificar los errores, las funciones semióticas permiten un refinamiento del análisis de los mismos que conduce a una visión más detallada del significado personal manifestado por los alumnos sobre un objeto matemático. A continuación, se realiza una descripción del proceso de refinamiento mencionado.

\section{Metodología empleada para definir las funciones semióticas}

En cada uno de los trabajos que se presentarán como ejemplos hubo un proceso de definición de funciones semióticas involucradas. Al hacer un análisis retrospectivo sobre esos procesos, se hallaron regularidades que se describirán a continuación.

En todos los casos se comienza por identificar una situación problema que, recurrentemente, presenta dificultades para los estudiantes. Esto impone la necesidad de determinar o explicar dichas dificultades. Para ello es necesario realizar una radiografía de la situación problema mediante una descripción detallada de las prácticas matemáticas que requiere su resolución. Una configuración epistémica hace posible dicha descripción. En ella se detallan los objetos matemáticos primarios presentes. Para cada uno de esos objetos primarios se requiere, por parte del estudiante, una práctica matemática (operativa, discursiva etc.). Esta asociación entre objetos primarios es la que se modeliza a través de funciones 
semióticas.

El conjunto de funciones semióticas así definidas conforma una trama inicial, o provisoria, que permite hacer ostensibles las prácticas matemáticas necesarias para resolver el problema. Dicha trama puede ir refinándose en su nivel de detalle si esto fuera necesario.

Este entramado es una herramienta que permite el análisis de las configuraciones cognitivas de las producciones de los estudiantes. En dicho análisis se indaga si se evidencia el establecimiento de las funciones semióticas en juego. Para aquellas funciones que no se muestran establecidas se debe proceder a analizar si el estudiante no estableció ninguna función semiótica o estableció una errónea, respecto del objeto primario que está estudiando. En este punto, es necesario preguntarse si la discrepancia entre la función semiótica definida como correcta, y la efectivamente utilizada por el estudiante es suficiente para explicar su error en la resolución del problema. En los casos en los que no resulta suficiente, se desagrega la función en cuestión en funciones semióticas que tributan en la construcción de la función semiótica estudiada. Esto dará lugar a un análisis posterior de las configuraciones cognitivas, comparando con estas nuevas funciones semióticas.

A continuación, en la Figura 1, se presenta un esquema que sintetiza lo anteriormente detallado respecto del proceso de refinamiento del entramado de las funciones semióticas. 


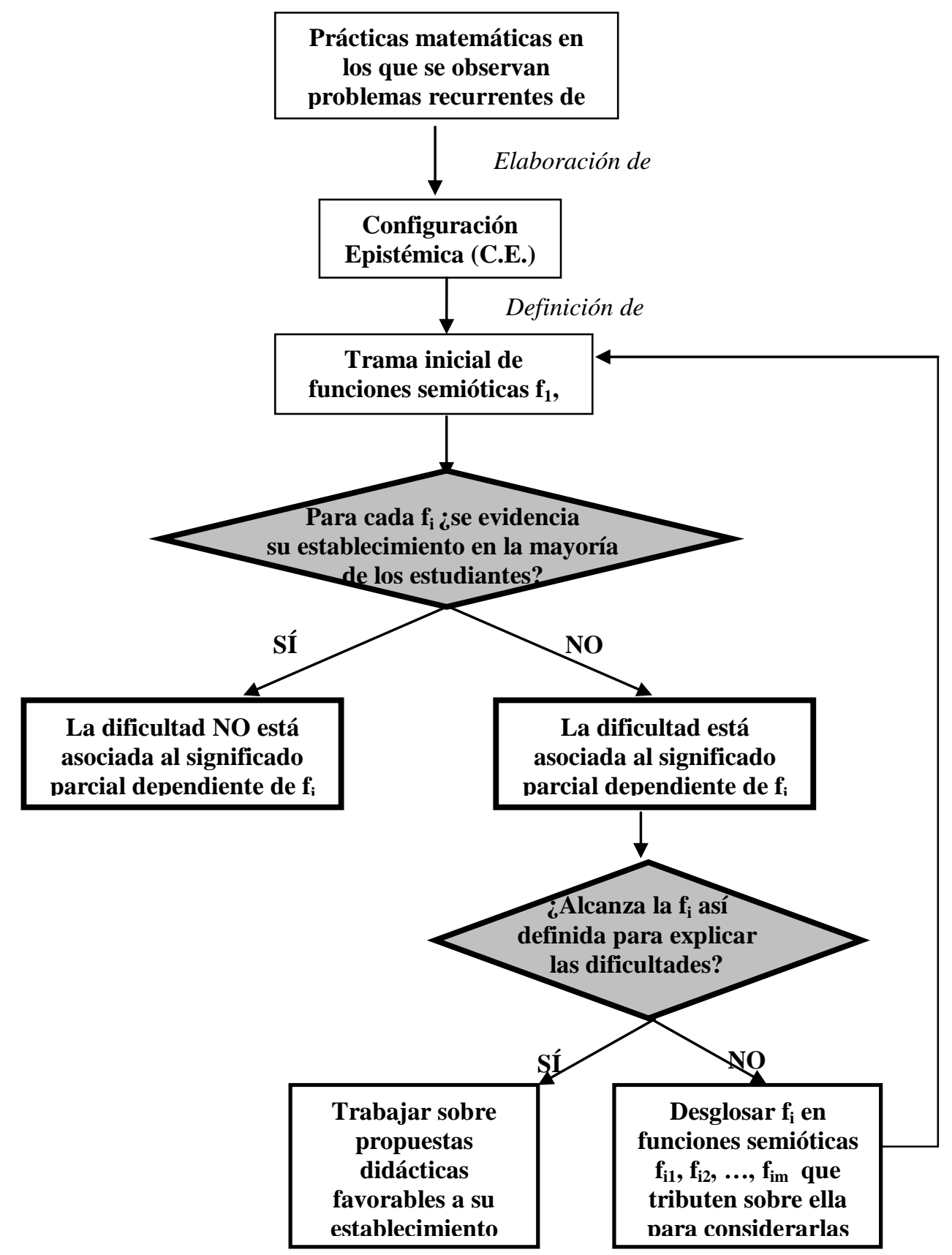

Figura 1- Proceso de definición y refinamiento de funciones semióticas. Fuente: desarrollado por los autores

\section{Ejemplos de aplicación de funciones semióticas}

A continuación se presentan los cuatro ejemplos, mencionados en la introducción, del uso de Funciones Semióticas como herramienta metodológica para analizar problemas de aprendizaje. En todos los casos, se trabajó con las producciones de alumnos que se encontraban cursando materias básicas de las carreras de ingeniería de la Universidad Nacional de Mar del Plata, Argentina. 


\subsection{Funciones semióticas asociadas a la resolución de un problema de optimización ${ }^{3}$}

Este ejemplo muestra el uso de las funciones semióticas para describir las dificultades para resolver problemas de optimización de un grupo estudiantes de un primer curso de Análisis Matemático, con el fin de extraer conclusiones que contribuyan a mejorar la enseñanza de este tema. El enunciado del problema $(\mathrm{P})$ con el que se trabajó es:

Determinar la mayor área que puede encerrar un rombo cuyo lado mide 1 metro. (Recordar que un rombo tiene todos sus lados congruentes y su área es la mitad del producto de sus diagonales).

En la Figura 2 se muestran las Funciones Semióticas asociadas a las prácticas con errores más frecuentes en la resolución del problema anteriormente enunciado.

Antecedente

\begin{tabular}{|c|c|c|}
\hline Condición del problema & $\mathrm{F}_{1}$ & $\begin{array}{l}\text { Establecimiento de la relación entre las variables de } \\
\text { la función a maximizar }\end{array}$ \\
\hline $\begin{array}{llll}\begin{array}{l}\text { Función área en dos } \\
\text { variables }\end{array} & & \\
\end{array}$ & $\mathrm{F}_{2}$ & $\begin{array}{l}\text { Identificación de la función área a maximizar en dos } \\
\text { variables }\end{array}$ \\
\hline $\begin{array}{l}\text { Función a maximizar en } \\
\text { una variable }\end{array}$ & $\mathrm{F}_{3}$ & $\begin{array}{l}\text { Re-expresión en función de una sola variable la } \\
\text { función área }\end{array}$ \\
\hline Derivada de la función & $\mathrm{F}_{4}$ & $\begin{array}{l}\text { Obtención de la función a partir de la aplicación de } \\
\text { reglas de derivación a la función a maximizar }\end{array}$ \\
\hline Punto crítico & $\mathrm{F}_{5}$ & $\begin{array}{l}\text { Obtención del valor que hace cero la derivada } \\
\text { primera o que no la define. }\end{array}$ \\
\hline Posible extremo relativo & $\mathrm{F}_{6}$ & $\begin{array}{l}\text { Punto crítico que se verifica como extremo con } \\
\text { algún criterio }\end{array}$ \\
\hline $\begin{array}{l}\text { Criterios para determinar si } \\
\text { un valor crítico es un } \\
\text { extremo relativo }\end{array}$ & $\mathrm{F}_{7}$ & $\begin{array}{l}\text { Determinación de la variación del signo de la } \\
\text { derivada primera en un entorno del punto crítico o } \\
\text { signo de la derivada segunda en dicho punto o } \\
\text { variación de la función en un entorno }\end{array}$ \\
\hline
\end{tabular}

Figura 2 - Funciones semióticas intervinientes en P.

Fuente: Baccelli et al. (2013)

La función semiótica $F_{1}$ es del tipo situacional-conceptual. Se consideró necesario desagregarla en sub-funciones que son detalladas en la Figura 3. Dichas funciones permitieron explicar, con detalle, las dificultades que se originan en el planteo del problema mencionado.

\begin{tabular}{|l|l|}
\hline \multirow{2}{*}{$\mathrm{F}_{1}$} & Rombo \\
\cline { 2 - 3 } & $\begin{array}{l}\text { Diagonales de un } \\
\text { Rombo }\end{array}$ \\
\cline { 2 - 2 } & $\begin{array}{l}\text { Teorema de } \\
\text { Pitágoras }\end{array}$ \\
\hline
\end{tabular}

\begin{tabular}{|c|c|}
\hline $\mathrm{F}_{11}$ & Cuadrilátero que tiene todos sus lados congruentes \\
\hline $\mathrm{F}_{12}$ & Diagonales perpendiculares que se cortan en su punto medio \\
\hline $\mathrm{F}_{13}$ & $\begin{array}{l}\text { La suma del cuadrado de los catetos de un triángulo } \\
\text { rectángulo es igual al cuadrado de la hipotenusa }\end{array}$ \\
\hline
\end{tabular}

Figura 3 - Funciones semióticas desagregadas a $\mathrm{F}_{1}$ en $\mathrm{P}$.

Fuente: Baccelli et al. (2013)

Las funciones $\mathrm{F}_{2}$ y $\mathrm{F}_{3}$ son del tipo proposicionales - actuativas. La función semiótica $\mathrm{F}_{3}$

\footnotetext{
${ }^{3}$ Baccelli, S.; Anchorena, S.; Figueroa, S.; Prieto G. (2013).
} 
muestra la dificultad de los alumnos de determinar la función a maximizar en una variable, incluso en aquellos que tienen establecidas las funciones $F_{1} y_{2} F_{2}$ La función $F_{4}$ es del tipo actuativa, mientras que la función $\mathrm{F}_{5}$ es del tipo conceptual. La función $\mathrm{F}_{6}$ corresponde a una función argumentativa y la función $\mathrm{F}_{7}$ además de ser argumentativa es actuativa.

\subsection{Funciones semióticas ligadas a distintas representaciones de números complejos ${ }^{4}$}

Este ejemplo muestra el uso de las funciones semióticas para analizar las dificultades y errores que se generan cuando los alumnos usan las representaciones aritmético-algebraica y geométrica-vectorial de los números complejos, en un grupo de estudiantes de un primer curso de Álgebra.

Se propusieron dos ejercicios a los estudiantes. En el primero de ellos se expone una proposición cuyos elementos están representados en lenguaje aritmético-algebraico. Su valor de verdad debe ser determinado por los alumnos a partir de la obtención de la parte imaginaria de la potencia de un número complejo expresado en forma binómica. Su enunciado es:

Determinar si es verdadera o falsa la siguiente expresión: Si $z=2-2 \sqrt{3} i$ i entonces $\operatorname{Im}\left(z^{21}\right)=1$.

Para analizar el significado algebraico que los estudiantes tenían construido, se definieron dos funciones semióticas presentes en ese proceso de significación. Las mismas se representan en la Figura 4.

\section{Antecedente}

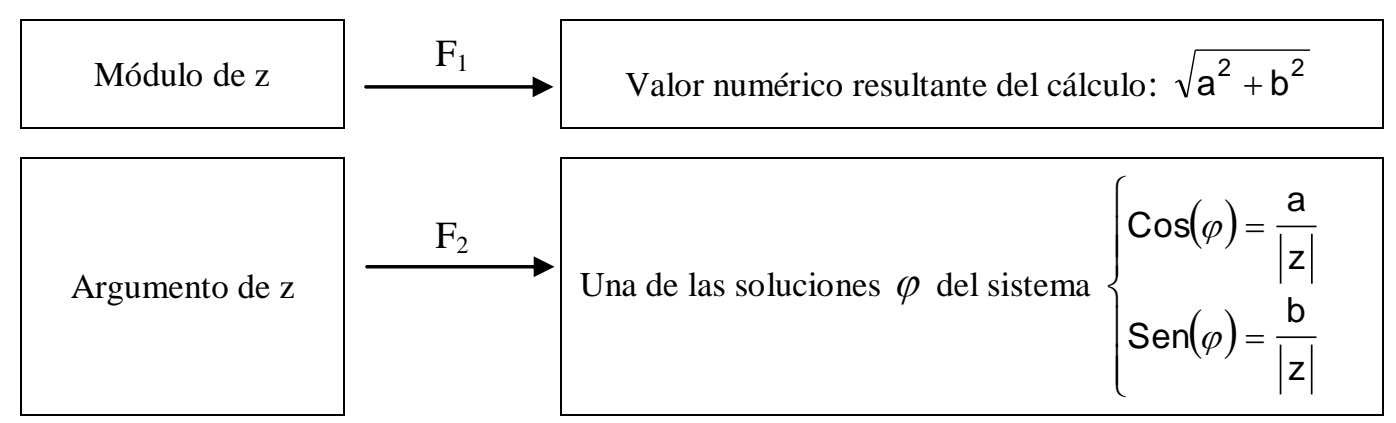

Figura 4 - Algunas funciones semióticas del Ejercicio 1. Fuente: Distéfano et al. (2012)

Las funciones $F_{1}$ y $F_{2}$ aluden, respectivamente, a significados actuativos (en el sentido que su contenido es una acción u operación, y en este caso, corresponde a un algoritmo o procedimiento) del módulo y del argumento de un número complejo $\mathrm{z}=\mathrm{a}+$ bi. Dichos significados actuativos están asociados a la representación aritmético-algebraica del mismo.

\footnotetext{
${ }^{4}$ Distéfano, M.L.,Aznar, M.A., Pochulu, M. (2012).
} 
Por consiguiente, puede considerarse que, para la correcta resolución de este ejercicio, se requiere haber construido un significado actuativo aritmético-algebraico ligado a esta forma de representación de los números complejos.

En el segundo ejercicio se proporciona la información en el registro gráfico. El enunciado es:

A la derecha figura la representación de los números complejos del

conjunto $B=\{z \in C,|z-4 i|=\sqrt{8}\}$.

a) Todos los números complejos del conjunto $B$ ¿tienen el mismo módulo? ¿Por qué?

b) ¿Entre qué valores varían los argumentos de los números complejos del conjunto B?

La resolución del inciso a) de este ejercicio puede hacerse utilizando únicamente herramientas del significado aritmético-algebraico, obteniendo elementos particulares del conjunto B y comparando sus módulos. Sin embargo, las mismas resultan insuficientes para la resolución del segundo inciso, que requiere la determinación de elementos de valores extremos de argumento del conjunto B y esto sólo puede realizarse a partir de la interpretación gráfica y geométrico-vectorial del mismo. Considerando que las herramientas del significado geométrico son las más viables para que los alumnos resuelvan ambos incisos, fueron definidas las funciones semióticas que se presentan a continuación (Figura 5).

Antecedente

\begin{tabular}{|c|c|c|}
\hline Número complejo z & $\mathrm{F}_{3}$ & Vector con origen en $(0,0)$ y extremo en su afijo $(a, b)$ \\
\hline Módulo de z & $\mathrm{F}_{4}$ & Distancia del afijo al origen o longitud del vector \\
\hline Argumento de $\mathrm{z}$ & $\mathrm{F}_{5}$ & $\begin{array}{l}\text { Ángulo con lado inicial en el semieje positivo de las } \\
\text { abscisas y lado final en la semirrecta que contiene al } \\
\text { vector asociado al número complejo. }\end{array}$ \\
\hline
\end{tabular}

Figura 5 - Algunas funciones semióticas del Ejercicio 2

Fuente: Distéfano et al. (2012)

Las funciones $\mathrm{F}_{3}, \mathrm{~F}_{4}$ y $\mathrm{F}_{5}$ hacen referencia al significado conceptual (en el sentido que se ha tenido en cuenta sólo un objeto primario del conjunto de prácticas: concepto, estableciéndose una correspondencia semiótica de tipo conceptual) asociado a la representación geométrica-vectorial de un número complejo $\mathrm{z}=\mathrm{a}+\mathrm{bi}$, donde los significados de módulo y argumento se derivan del significado de vector. Esto condiciona que la función semiótica $\mathrm{F}_{3}$ pueda establecerse sí, y solamente si, las funciones $\mathrm{F}_{4}$ y $\mathrm{F}_{5}$ son construidas. 
Puede considerarse que, para la correcta resolución de este ejercicio, es necesario haber construido un significado conceptual geométrico de los números complejos asociado a esta forma de representación, lo cual implica haber establecido estas tres funciones semióticas.

\subsection{Funciones semióticas presentes en el proceso de significación de algunos símbolos $\operatorname{algebraicos}^{5}$}

En este ejemplo se muestra el uso de algunas funciones semióticas para describir y caracterizar el proceso de construcción de significados de algunos símbolos algebraicos. Se consideraron las representaciones semióticas que involucran los símbolos de pertenencia, inclusión, conjunción, disyunción y cuantificadores.

Para analizar el significado que le otorgan los estudiantes a los símbolos se diseñaron actividades partiendo de la idea de que el significado de un símbolo está ligado a tres elementos: su identificación, la estructura semiótica de las expresiones en las que está presente y el valor de verdad de las mismas. Esto dio lugar a la definición de tres funciones semióticas presentes en el proceso de significación de todos los símbolos analizados.

La primera de ellas $\left(\mathrm{F}_{1}\right)$ vincula la expresión del símbolo con su expresión coloquial o denominación. La segunda $\left(\mathrm{F}_{2}\right)$ se establece entre la denominación mencionada y la estructura determinada por la sintaxis de la representación. Esa sintaxis involucra tanto el orden de los elementos como los roles jugados por ellos. La tercera función semiótica $\left(\mathrm{F}_{3}\right)$, es la que relaciona la proposición en la que está presente el símbolo, con su valor de verdad, el cual depende también de los significados de los operandos involucrados.

Como ejemplo, se presentan en la Figura 6 estas funciones semióticas para el caso particular del símbolo de pertenencia.

Antecedente
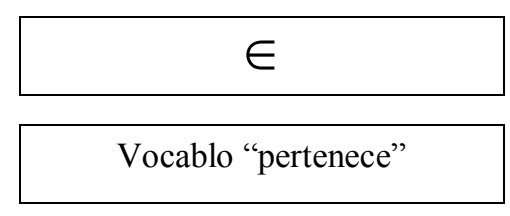

Elemento $\in$ conjunto

Figura 6 - Funcio

\section{Consecuente}

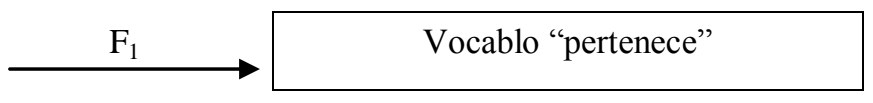

Elemento $\in$ conjunto

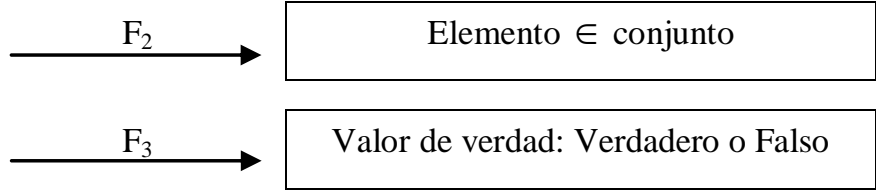

Fuente: Distéfano et al. (2014)

\footnotetext{
${ }^{5}$ Distéfano, M.L.; Aznar, M.A.; Pochulu, M. (2014).
} 


\subsection{Funciones semióticas asociadas a la resolución de problemas bayesianos ${ }^{6}$}

Este ejemplo muestra el uso de funciones semióticas que contribuyen a describir las dificultades en la resolución de problemas bayesianos en un grupo estudiantes de Estadística, con la finalidad de extraer conclusiones que favorezcan el aprendizaje y la enseñanza de la probabilidad condicional y del Teorema de Bayes.

El Problema ${ }^{7}$, cuyo enunciado se presenta a continuación, es el instrumento utilizado para evaluar las funciones semióticas involucradas.

Problema: Un taxi se vio implicado en un accidente nocturno con choque y huida posterior. Hay dos compañías de taxis en la ciudad, la Verde y la Azul. El $85 \%$ de los taxis de la ciudad son Verdes y el $15 \%$ Azules. Hubo un testigo del accidente. El tribunal comprobó la fiabilidad del testigo bajo las mismas circunstancias que había la noche del accidente y llegó a la conclusión de que el testigo identificaba correctamente cada uno de los colores en el $80 \%$ de las ocasiones y fallaba en el $20 \%$. Sabiendo que el testigo identificó el taxi como azul. ¿Cuál es la probabilidad de que el taxi implicado en el accidente fuera en efecto Azul?

En la Figura 7 se muestran Funciones Semióticas asociadas a la resolución del problema propuesto.

Antecedente

Consecuente

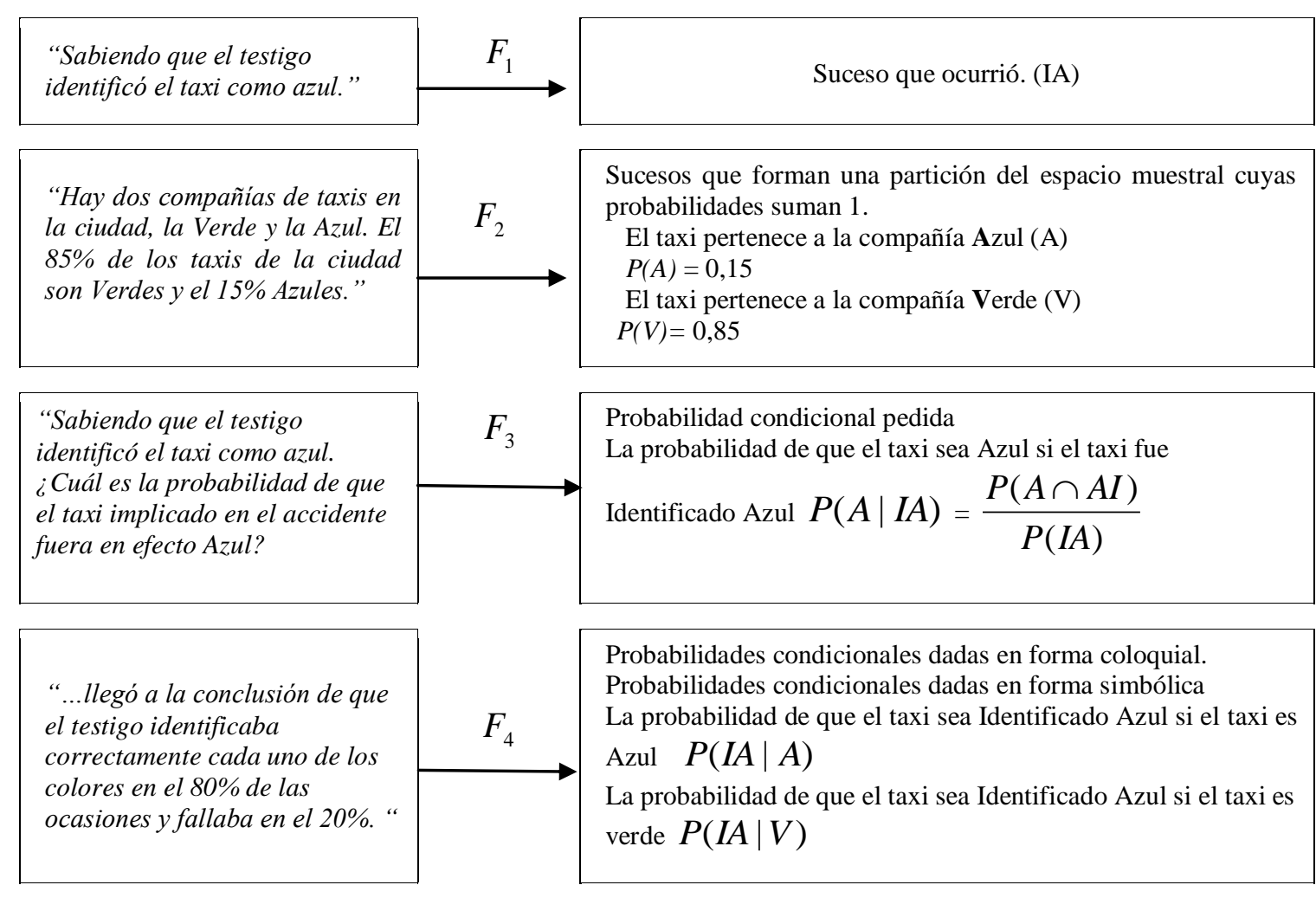

\footnotetext{
${ }^{6}$ Figueroa, S.; Anchorena, S.; Distéfano, M. L. (2012).

${ }^{7}$ TVERSKY, A. y KAHNEMAN, D. (1982, citado en DÍAZ; DE LA FUENTE, 2007a).
} 


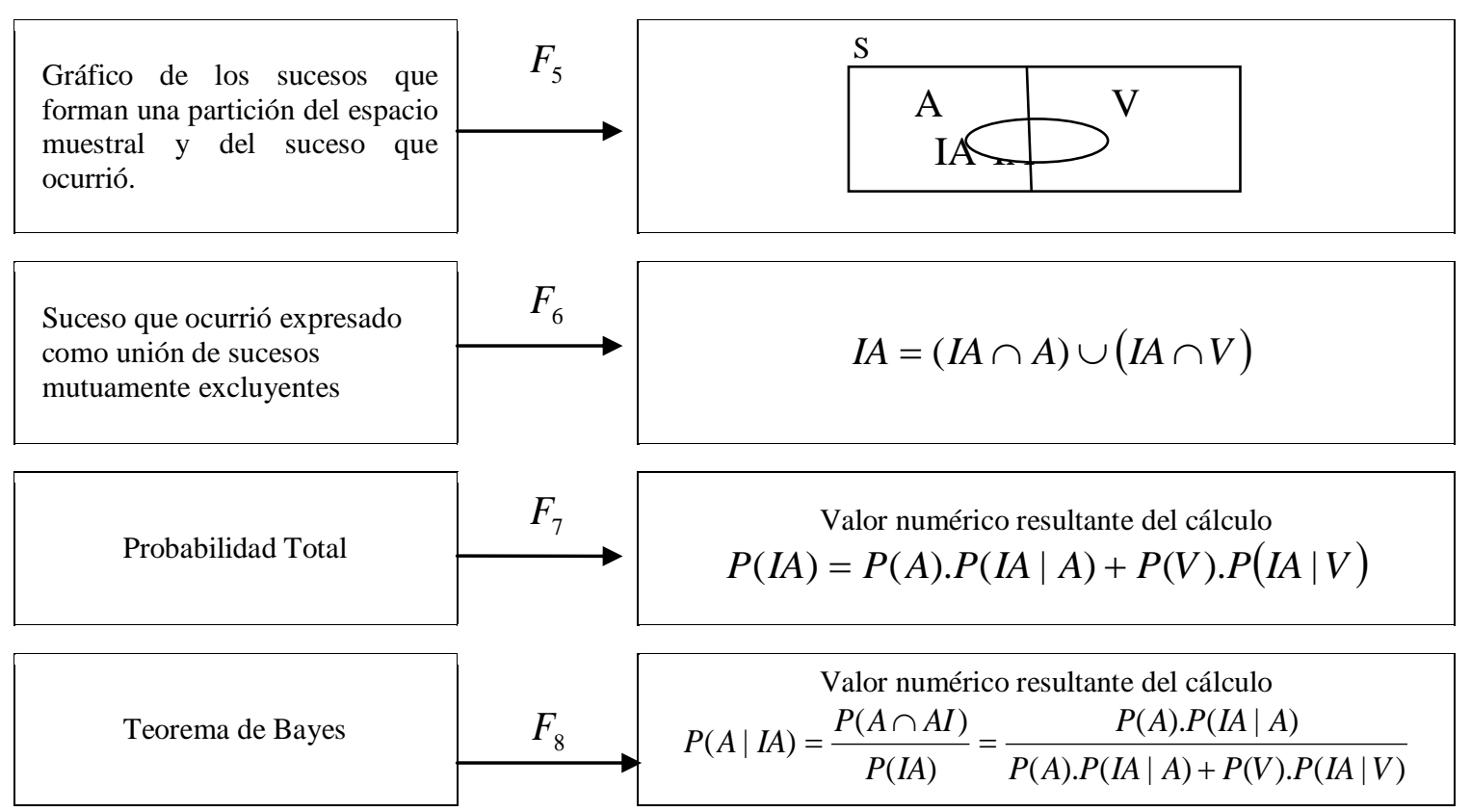

Figura 7 - Funciones Semióticas presentes en la resolución del problema Fuente: Figueroa et al. (2012)

Las funciones $F_{1}, F_{2}, F_{3}$ y $F_{4}$ aluden a significados situacionales-lingüísticos en el sentido que su contenido está vinculado a la identificación de los datos de un problema con los sucesos involucrados. Se establece, para cada caso, una función semiótica con el suceso que ocurrió, los otros sucesos que intervienen y sus probabilidades asociadas, simples y condicionales, respectivamente. Para la visualización del espacio muestral con cada suceso involucrado se establece la función semiótica $F_{5}$ que se refiere al significado dado por la representación gráfica del espacio muestral con dichos sucesos.

Con la función semiótica $\mathrm{F}_{6}$ se efectúa la correspondencia lingüística entre el suceso que ocurrió y la unión de sucesos mutuamente excluyentes, dando el punto de partida a la argumentación de la fórmula de la probabilidad total y luego a la del Teorema de Bayes, una vez que se establecen las funciones semióticas $\mathrm{F}_{7} \mathrm{y}_{8}$.

A partir de estas funciones semióticas se definieron los pasos para la resolución de este tipo de problemas. Esto permitió definir una metodología destinada a favorecer en los estudiantes su abordaje, que se sintetiza en los siguientes pasos:

1) La detección, en el problema, del suceso condicionante o principal A. Función situacional lingüística $(\mathrm{F} 1)$.

2) La identificación de los sucesos simples cuyas probabilidades suman uno. Función situacional lingüística $(\mathrm{F} 2)$.

3) La identificación de la probabilidad condicional pedida. Función situacional lingüística $(\mathrm{F} 3)$.

4) La identificación de las otras probabilidades condicionales en el problema. Función 
situacional lingüística (F4).

5) La representación gráfica del suceso condicionante $A$. Función linguística (F5).

6) La representación simbólica del suceso $A$ como unión de sucesos mutuamente excluyentes. Función conceptual linguística (F6).

7) El cálculo de la probabilidad total del suceso principal. Función actuativa argumentativa (F7).

8) La aplicación de la fórmula del teorema de Bayes al reemplazar la probabilidad pedida con el cálculo previo de la probabilidad total. Función actuativa argumentativa (F8).

El conjunto de funciones semióticas de este proceso de instrucción fue construido sobre la base del análisis de los errores más frecuentes que cometen los alumnos al resolver este tipo de problemas. El Cuadro 2 muestra esta asociación como una herramienta para el tratamiento de estos errores descriptos por Díaz y De La Fuente (2007b).

\begin{tabular}{|c|c|c|c|c|c|c|}
\hline & $\begin{array}{c}\text { Confusión } \\
\text { de } \\
\mathrm{P}\left(\mathrm{B}_{\mathrm{B}} / \mathrm{A}\right) \\
\text { con } \\
\mathrm{P}\left(\mathrm{A} / \mathrm{B}_{\mathrm{i}}\right)\end{array}$ & $\begin{array}{c}\text { Confusión de } \\
\mathrm{P}\left(\mathrm{A} / \mathrm{B}_{\mathrm{i}}\right) \\
\mathrm{Con} \\
\mathrm{P}\left(\mathrm{B}_{\mathrm{i}}\right)\end{array}$ & $\begin{array}{l}\text { Confusión de } \\
\left.\text { P(A/B } \mathrm{B}_{\mathrm{i}}\right) \\
\text { con } \\
\mathrm{P}\left(A \cap B_{i}\right)\end{array}$ & $\begin{array}{l}\text { Errores } \\
\text { en la } \\
\text { partición } \\
\text { de S }\end{array}$ & $\begin{array}{c}\text { Error en la } \\
\text { aplicación } \\
\quad \text { de la } \\
\text { Fórmula de } \\
\text { la P.Total }\end{array}$ & $\begin{array}{c}\text { Error en la } \\
\text { aplicación } \\
\text { de la } \\
\text { fórmula del } \\
\text { T. de } \\
\text { Bayes }\end{array}$ \\
\hline $\begin{array}{l}\mathbf{F}_{1} \text { Asigna la correspondencia } \\
\text { entre los datos del problema con } \\
\text { la identificación del suceso } \\
\text { condicionante A }\end{array}$ & $\mathbf{X}$ & & & & & \\
\hline $\begin{array}{l}\mathbf{F}_{2} \text { Establece la identificación } \\
\text { de los sucesos que forman una } \\
\text { partición del espacio muestral } \mathrm{S} \text {, } \\
\text { los sucesos } \mathrm{Bi}\end{array}$ & & $\mathbf{X}$ & & $\mathbf{X}$ & & \\
\hline $\begin{array}{l}\mathbf{F}_{3} \text { Establece la identificación } \\
\text { entre los datos del problema y la } \\
\text { probabilidad condicional } \\
\text { pedida. }\end{array}$ & $\mathbf{X}$ & & $\mathbf{X}$ & & & \\
\hline $\begin{array}{l}\mathbf{F}_{4} \text { Asigna la correspondencia } \\
\text { entre las probabilidades } \\
\text { condicionales dadas en el } \\
\text { problema con con las } \\
\text { probabilidades condicionales } \\
\text { en forma simbólica. }\end{array}$ & $\mathbf{X}$ & & $\mathbf{X}$ & & & \\
\hline $\begin{array}{l}\mathbf{F}_{5} \text { Asigna un gráfico con los } \\
\text { sucesos que forman una } \\
\text { partición del espacio muestral y } \\
\text { el suceso A }\end{array}$ & & & & $\mathbf{X}$ & $\mathbf{X}$ & $\mathbf{X}$ \\
\hline $\begin{array}{l}\mathbf{F}_{6} \text { Asigna al suceso A la } \\
\text { unión de sucesos mutuamente } \\
\text { excluyentes. }\end{array}$ & & & & & $\mathbf{X}$ & $\mathbf{X}$ \\
\hline $\begin{array}{l}\mathbf{F}_{7} \text { Establece el valor numérico } \\
\text { resultante del cálculo de la } \\
\text { probabilidad Total }\end{array}$ & & & & & $\mathbf{X}$ & \\
\hline
\end{tabular}

Cuadro 2 - Funciones semióticas asociadas a los errores más frecuentes en la resolución de problemas bayesianos

Fuente: Figueroa et al. (2012) 


\section{Consideraciones finales}

En las secciones anteriores se ha ejemplificado y descripto la utilización de funciones semióticas, como herramientas teóricas y metodológicas del EOS. Dentro de este marco teórico se entiende el aprendizaje como el proceso por el cual un estudiante hace suyo un conjunto de significados, lo que hace relevante el estudio de los mismos. En este contexto, los significados comprenden prácticas operativas y discursivas en la resolución de determinado tipo de problemas. Para una mirada profunda sobre dichas prácticas se las puede estructurar o desglosar definiendo funciones semióticas que el estudiante tiene que establecer para poder realizarlas. El establecimiento deficiente de funciones semióticas se manifiesta en prácticas calificadas como erróneas. Errores asociados al incorrecto establecimiento de algunos tipos de funciones semióticas pueden ubicarse en categorías de algunos de los estudios de errores como los realizados por Radatz o Mavshovitz-Hadar, Zaslavksy e Invar.

En los antecedentes y ejemplos presentados puede observarse que las funciones semióticas permitieron:

- Hacer visible el nivel de complejidad de una determinada práctica matemática, considerando los objetos primarios y las facetas duales que el estudiante debe vincular.

- Anticipar conflictos semióticos potenciales en trayectorias y materiales didácticos, que se podrían producir por no tener en cuenta dicha complejidad.

- Evaluar detalladamente prácticas matemáticas de estudiantes, detectando disparidad de significados.

- Favorecer el rediseño de secuencias didácticas, contemplando las complejidades y conflictos detectados.

En este trabajo se expuso una forma sistemática de uso de las funciones semióticas para optimizar el aprovechamiento de su potencial como instrumento metodológico. Esta sistematización da cuenta de los pasos a seguir en el análisis de errores recurrentes en relación a un determinado objeto matemático, refinando el entramado de funciones semióticas de acuerdo a los requerimientos de la resolución del problema.

El potencial de este tipo de análisis radica en su aplicación para el diagnóstico y abordaje de distintos tipos de errores asociados a prácticas matemáticas que definen el significado de un objeto matemático. Sin embargo, debe señalarse que dicho análisis está localizado en objetos matemáticos particulares. El empleo de esta herramienta en el estudio de la enseñanza y aprendizaje de variados objetos matemáticos podría dar lugar a investigaciones más generales en el futuro. 


\section{Referencias}

ABRATE, R.; POCHULU, M.; VARGAS, J. Errores y dificultades en Matemática: análisis de causas y sugerencias de trabajo. Universidad Nacional de Villa María, Buenos Aires, 2006.

BACCELLI, S; ANCHORENA, S.; FIGUEROA, S.; PRIETO G. Análisis de significado para mejorarlos aprendizajes en problemas de optimización. In: CONGRESO IBEROAMERICANO DE EDUCACIÓN MATEMÁTICA, 7., 2013, Montevideo. Actas. Montevideo: SEMUR, 2013. p. 30793088.

BORASSI, R. Exploring Mathematics through the Analysis of Errors. For the Learning of Mathematics, Fredericton, v. 7, p. 2-9, 1987.

CHACÓN, R. Análisis del proceso de instrucción del Algebra abstracta en la Universidad Nacional abierta, desde una perspectiva Semiótico-Didáctica. 2006. 141F. Tesis de Maestría Universidad Nacional Abierta, Barinas, Venezuela, 2006. Disponible en: <http://biblo.una.edu.ve/docu.7/bases/marc/texto/t10161.pdf >. Acceso en: 14 abr. 2013.

CONTRERAS, A.; FONT, V.; LUQUE, L.; ORDOÑEZ, L. Algunas aplicaciones de la teoría de las funciones semióticas a la didáctica del análisis infinitesimal. Recherches en Didactique des Mathématiques, Grenoble, v. 25, n. 2, p. 151-186, 2005. Disponible en: $<$ http://webs.ono.com/vicencfont/RDMfinal.pdf>. Acceso en: 27 jul. 2013.

CONTRERAS, A.; ORDOÑEZ, L. Complejidad ontosemiótica de un texto sobre la introducción a la integral definida. Revista Latinoamericana de Matemática Educativa, México, v. 9, n. 1, p. 65-84, 2006. Disponible en: <http://www.scielo.org.mx/pdf/relime/v9n1/v9n1a4.pdf>. Acceso en: 14 ago. 2013.

DÍAZ, C.; DE LA FUENTE, I. Validación de un cuestionario de razonamiento probabilístico condicional. Revista Electrónica de Metodología Aplicada, Principado de Asturias, v. 2, n. 1, p. 115. 2007a. Disponible en: 〈http://www.psico.uniovi.es/REMA/v12n1/a1.pdf>. Acceso en: 24 oct. 2012.

DÍAZ, C.; DE LA FUENTE, I. Dificultades en la resolución de problemas que involucran el Teorema de Bayes. Un estudio exploratorio en estudiantes de psicología. Educación Matemática, Granada, v. 2, n. 18, p.75-94. 2007b. Disponible en: < http://www.ugr.es/ batanero/pages/didacticaproba bilidad.html \#Probabilidad_Condicional>. Acceso en: 30 jun. 2015.

DISTÉFANO, M. L.; AZNAR, M. A.; POCHULU, M. Errores asociados a la representación geométrica-vectorial de números complejos: un análisis ontosemiótico. Revista Iberoamericana de Educación Matemática UNIÓN. España, n. 30, p. 61-80, 2012. Disponible en: <http://www.fisem. org/web/union/images/stories/30/Archivo_9_de_volumen_30.pdf>. Acceso en: 28 mar. 2014.

DISTÉFANO, M. L.; AZNAR, M. A.; POCHULU, M. Niveles de construcción de significado de algunos símbolos matemáticos. Acta Latinoamericana de Educación Matemática - ALME, 27, 2014, Coalco. Actas, Coalco, 2014. p. 209-218. Disponible en: <http://www.clame.org.mx/documentos/ alme27.pdf>. Acceso en: 29 ago. 2014

FIGUEROA, S.; ANCHORENA, S.; DISTÉFANO, M. L. Valoración de la Idoneidad Epistémica y Cognitiva de un Proceso de Instrucción en la Resolución de Problemas Bayesianos. Bolema, Rio Claro, v. 28, n. 48, p. 169-190, 2012. Disponible en: <http://www.periodicos.rc.biblioteca.unesp .br/index.php/bolema/article/view/6676/6079/>. Acceso en: 14 may. 2014.

FIGUEROA, S. BACCELLI, S. PRIETO, G. MOLER, E. Funciones semióticas asociadas a los errores más frecuentes en la resolución de problemas bayesianos. In: REUNIÓN DE EDUCACIÓN 
MATEMÁTICA - REM, 35, 2012, Córdoba. Actas, Córdoba, 2012. Disponible en: $<$ http://www2.famaf.unc.edu.ar/rev_edu/>. Acceso en: 14 may. 2014.

FONT, V. Una organización de los programas de investigación en Didáctica de las Matemáticas. Revista EMA, Santa Fe de Bogotá, v. 7, n. 2, p. 127-170, 2002.

GODINO, J.; BATANERO, C. Significado institucional y personal de los objetos matemáticos. Recherches en Didactique des Mathématiques, v. 14, n. 3, p. 325-355, 1994. Disponible en: <http://rdm.penseesauvage.com/RDM-Vol-14-3.html>. Acceso en: 24 oct. 2012.

GODINO, J. D. Teoría de las funciones semióticas: Un enfoque ontológico-semiótico de la cognición e instrucción matemática. Granada: Servicio de reprografía de la Facultad de Ciencias, 2003. Disponible en: 〈http://www.ugr.es/ jgodino/funciones-semioticas/monografiatfs.pdf>. Acceso en: 24 abr. 2013.

GODINO, J. D.; BATANERO, C.; ROA, R. An onto-semiotic analysis of combinatorial problems and the solving processes by university students. Educational Studies in Mathematics, Dordretch, v. 60, n. 1, p. 3-36, 2005.

GODINO, J. D.; CONTRERAS, A.; FONT, V. Análisis de procesos de instrucción basado en el enfoque ontológico-semiótico de la cognición matemática. Recherches en Didactiques des Mathematiques, Grenoble, v. 26, n. 1, p. 39-88, 2006.

GODINO, J.; BATANERO, C.; FONT, V. Un enfoque ontosemiótico del conocimiento y la instrucción matemática. Departamento de Didáctica de la Matemática. Universidad de Granada. 2009. Disponible en: <http://www.ugr.es/ jgodino/funciones-semioticas/sintesis_eos_10marzo 08.pdf.>. Acceso en: 12 ago. 2011.

POCHULU, M. D. Análisis y categorización de errores en el aprendizaje de la matemática en alumnos que ingresan a la universidad. Revista Iberoamericana de Educación, v. 35, n. 4, p.1-15. 2004.

Disponible en: <www.campusoei.org/revista/deloslectores/849Pochulu.pdf>. Acceso en: 30 ago. 2011.

POCHULU, M. Enfoque Ontosemiótico del Conocimiento y la Instrucción Matemática. In:

POCHULU, M. Y.; RODRÍGUEZ, M. (Coord.). Educación Matemática: Aportes a la formación docente desde distintos enfoques teóricos. Villa María: Editorial Universitaria Villa María, p. 63-89, 2012.

RADATZ, H. Students' Errors in the Mathematical Learning Process: a Survey. For the Learning of Mathematics v. 1, n. 1, p. 16-20, 1980.

RICO, L. Errores en el aprendizaje de la Matemática. In: KILPATRICK, J.; GÓMEZ, P.; RICO LUIS, L. (Coord.). Educación Matemática, México: Grupo Editorial Iberoamérica, 1995. p. 69-108.

ROJAS GARZÓN, P. Articulación y cambios de sentido en situaciones de tratamiento de representaciones simbólicas de objetos matemáticos. 2012. 128F. Tesis de Doctorado - Facultad de Ciencias y Educación, Universidad Distrital Francisco José de Caldas, Bogotá, 2012. Disponible en: <http://www.dm.unibo.it/rsddm/it/Phd/Rojas\%20Garzon/Tesis\%20Pedro\%20Rojas.pdf >. Acceso en: 14 jun. 2014.

TAMAYO, G.; SOLANO, Á.; TORRES, P.; ORTIZ, J.; FERNÁNDEZ, A. Tecnología digital, actos y procesos semióticos en la definición de límite funcional de Weierstrass. In: ENCUENTRO COLOMBIANO DE MATEMÁTICA EDUCATIVA, 9, 2008, Valledupar, Colombia. Memorias del 9no Encuentro Colombiano de Matemática Educativa. Bogotá: García, G., 2008. p. 57-63. Disponible en: < http://asocolme.org/publicaciones-asocolme/memorias-ecme>. Acceso en: 26 abr. 2016. 
Submetido em Outubro de 2014. Aprovado em Abril de 2015. 